\title{
Article Type: Articles
}

\section{$\bar{\circ}$ \\ - The Dynamics of Adolescent Depression: an \\ Instrumental Variable Quantile Regression with Fixed \\ Effects Approach}

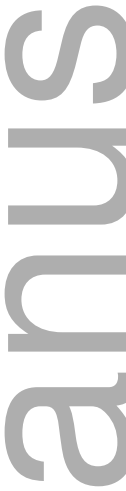

\author{
Paul Contoyannis *
}

Jinhu $\mathrm{Li}^{\dagger}$

\begin{abstract}
* Department of Economics, McMaster University; Centre for Health Economics and Policy Analysis, McMaster University; contoyp@ mcmaster.ca

$\dagger$ Corresponding author: Melbourne Institute of Applied Economic and Social Research, The University of Melbourne; Address: Level 5, Faculty of Business and Economics Building, 111 Barry Street, Victoria 3010 Australia; Email: jinhu.li@unimelb.edu.au ; Telephone: +61-3-9035 3753; Fax: +61-3-8344 2111.
\end{abstract}

We thank Joāo Santos Silva, Alfia Karimova and participants for very helpful suggestions at the $3^{\text {rd }}$ Annual Health Econometrics Workshop, the $10^{\text {th }}$ Annual Meeting of the Canadian Health Economists' Study Group (CHESG), and the 2012 European Conference on Health Economics. We also thank Antonio Galvao and Roger Koenker for their valuable advice. Finally, we thank the Shared Hierarchical Academic Research Computing Network (SHARCNET) for granting us access to their network of high performance computers. This research was partly funded by a 2012 Early Career Researcher Grant from The University of Melbourne. None of the above bears any responsibility for the contents of this work.

This is the author manuscript accepted for publication and has undergone full peer review but has not been through the copyediting, typesetting, pagination and proofreading process, which may lead to differences between this version and the Version of Record. Please cite this article as doi: 10.1111/rssa.12225

This article is protected by copyright. All rights reserved 


\begin{abstract}
This paper employs a recently-developed instrumental variable approach for the estimation of dynamic quantile regression models with fixed effects to model the dynamics of health outcomes. Our proposed estimator not only allows us to control for individual-specific heterogeneity via fixed effects in the dynamic quantile regression framework, but also may reduce the bias that exists in conventional fixedeffects estimation of dynamic quantile regression models with small numbers of time periods. Using data on the children of the US National Longitudinal Survey of Youth 1979 cohort (CNLSY79), we examine the extent of true state dependence in youth depression conditional on unobserved individual heterogeneity and family socioeconomic status. Our results suggest that true state dependence in youth depression among the CNLSY79 respondents is very low and the observed positive association between previous and current depression is mainly due to time-invariant unobserved individual heterogeneity.
\end{abstract}

Keywords: adolescence, depression, dynamic quantile regressions, health dynamics, instrumental variables quantile regression, panel data models

JEL codes: I12, C23, C22

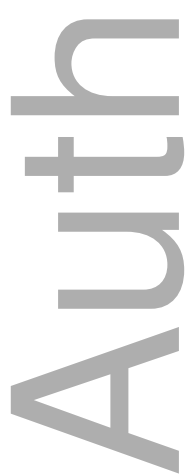

This article is protected by copyright. All rights reserved 


\section{Introduction}

An emerging literature in economics has documented the critical role of childhood health capital in shaping life-time social and economic success. Some studies have established the importance of childhood physical health (Case et al. 2005; Smith 2009; Currie et al. 2010), while others have demonstrated overshadowing consequences of childhood mental disorders and behavioural problems on future outcomes over the life cycle (see Currie and Stabile 2006; Contoyannis and Dooley 2010; Smith and Smith 2010; and Goodman et al 2011). This motivates our examination of health accumulation during the critical period of pre-adulthood. It is increasingly recognized that child development has the multi-stage nature where output in one stage and other inputs determine output in the next stage (Heckman 2012). This suggests that in order to understand the evolution of health during childhood, it is critical to model the health production in a dynamic framework where current health is determined by previous health and other contemporaneous characteristics. The empirical modeling of this process commonly involves estimating a specification that includes lagged dependent variables as regressors. The recent literature on health dynamics has found that it is often the case that some individuals experience persistently good or poor health and this persistence could be explained by three different mechanisms (see Jones et al 2012). Firstly, some observed individual characteristics can predispose them to poor health that lingers over time. Secondly, unobserved factors such as time preference, risk preference or non-cognitive abilities can have a long-standing influence on individuals' health. This suggests that certain unobserved personal attributes may lead to prolonged poor health over time. Thirdly, persistence can reflect the nature of certain illnesses-the experience of one episode leads to elevated risks of recurrence of the illness over time, conditional on observed and unobserved characteristics. This is termed true state dependence. Since they are very different causal mechanisms, it is important to separate true state dependence from observed characteristics and unobserved individual heterogeneity in the empirical modelling of health dynamics. 
In this study we empirically model the dynamics of depression during adolescence. This specific application is motivated by the fact that depression is one of the most prevalent mental health problems among adolescents (Merikangas et al. 2010). Moreover, depression in adolescence is associated with various adverse short-term and long-term consequences (see Fergusson et al. 2007). Descriptive evidence has shown that adolescents who experience depression often struggle with depression throughout their lives (Lewinsohn et al. 1999), and in many cases, early onset of depression predicts higher rates of recurrence of major depression (Greden 2001), and even more severe depression during adulthood (Weissman et al. 1999). Studies in biological psychiatry provide a clinical explanation for this phenomenon. Farb et al. (2011) suggest that among adult patients with clinically diagnosed depression the physiological changes in the brain chemistry caused by a first episode of depression are likely to affect their perception of risk and cause them to become hypersensitive to external threats. Therefore, they will have another episode of depression with smaller triggers than those that caused the first episode of depression. Although this persistent nature of depression has been examined among clinically diagnosed adult patients, little is known about the dynamics of depression in a general population of adolescents. The findings from clinically diagnosed samples may not be generalizable to a broader population. It is also recognized that the nature of mental disorders in young people are somewhat different from those of adults. From a societal perspective, therefore, it is important to identify social or family risk factors that determine the first episode of depression, and the risk factors associated with persistent depression among adolescents.

In this study we model the dynamics of depression among adolescents, aiming to address two questions: 1) How much persistence there is in depression among adolescents in a general population survey? 2) What are the underlying reasons for any observed persistence in youth depression? Using data on the children of the US National Longitudinal Survey of Youth 1979 cohort (CNLSY79), this paper quantifies the persistence of youth depression and examines the extent of true state dependence, 
conditional on unobserved individual heterogeneity and observed family socioeconomic status (SES).

Existing empirical studies on health dynamics have mostly relied on random effects dynamic conditional mean panel data models where the mean of current health is modelled as a function of lagged health and other characteristics (see e.g. Contoyannis and $\mathrm{Li}$ 2011, Jones et al 2012). The consistent estimation of state dependence using this estimator hinges on an appropriate model for initial conditions. More importantly, it is problematic to use dynamic conditional mean panel data models to account for heterogeneity, which is very likely to exist in the true state dependence and the effects of socioeconomic factors on health dynamics. In fact, based on the exiting studies the estimates of true state dependence and the effects of socioeconomic factors on health dynamics vary across different age distributions, study samples and data sets (Contoyannis et al. 2004b; Contoyannis and Li 2011; Ham et al. 2013; Hauck and Rice 2004; Repetto et al. 2004; Roy and Schurer 2013; Rushton et al. 2002; Strohschein 2005). Discrepancies in these studies could indicate the heterogeneity in the effects of these characteristics over the conditional distribution of the specific health outcome and regressors.

Heterogeneity can exist in intercepts only, or in both intercepts and coefficients. For the former case standard random or fixed effects estimators within the dynamic conditional mean estimation framework are problematic -- the standard fixed-effects estimator (based on OLS after first differencing) is inconsistent for short panels due to the inclusion of lagged dependent variables as regressors, while random effects estimators additionally require an appropriate model for initial conditions. Instrumental variable estimators within the dynamic conditional mean estimation framework, however, can be used which are independent of initial conditions and are consistent for fixed $\mathrm{T}$ (i.e. time periods) and large $\mathrm{N}$ (i.e. cross section of individuals) in both fixed and random effects models (see e.g. Hsiao (2003) Chapter 4). When heterogeneity exists in both intercepts and coefficients, which is very likely in our application, the situation 
is more problematic with the dynamic conditional mean estimation framework. With small $\mathrm{T}$ it is not feasible to compute ordinary (or generalized) least squares estimates for arbitrary distributions of heterogeneity in either fixed or random effects models if there are more regressors than time periods. While Bayesian approaches exist to estimate average effects for small $\mathrm{T}$, these focus on appropriately estimating average effects rather than exploring heterogeneity (see e.g. Hsiao (2003) Chapter 6.7). In this case, using an estimator appropriate for homogenous effects of covariates will produce a weighted average of heterogeneous effects. This implies that estimates from models not accounting for heterogeneous effects will vary depending on the distribution of the regressors in the sample, even when average effects are appropriately estimated.

Given the above limitations, we instead employ conditional quantile regression framework to account for potential heterogeneity in both intercepts and coefficients, allowing flexibly for heterogeneous effects of the covariates across the outcome distribution. Similarly random or fixed effects approaches can be used within the dynamic conditional quantile regression framework. There is a small recent literature on the estimation of dynamic random effect quantile regression models (e.g. Arellano and Bonhomme 2013). While this is an interesting alternative approach they require specification of the relationship between the individual effects and regressors and the initial conditions for point identification and consistent estimation with small $\mathrm{T}$. In order to avoid making strong assumptions, we use an instrumental variable (IV) approach based on Galvao (2011) for the estimation of dynamic quantile regression models with fixed effects. Specifically, our proposed estimator controls for individual-specific heterogeneity via fixed effects (instead of random effects) in the dynamic quantile regression framework. Analogous to that used by Anderson and Hsiao (1981) in the dynamic conditional mean context, lags of the dependent variable are used as instruments. This IV estimator effectively reduces the bias that exists in conventional fixed-effects estimation of dynamic quantile regression models. (See Galvao (2011) for Monte-Carlo evidence comparing the bias of the IV estimator and of conventional 
fixed-effects quantile regression estimator in the context of a continuous outcome variable.) To account for the nature of the dependent variable in our study, a discrete depression score, we also integrate this IV estimator with the 'jittering' approach suggested for estimating conditional quantile models for count data in a cross-sectional context (Machado and Santos Silva 2005).

Our study provides methodological contribution to the literature on the empirical modeling of health dynamics. Ours is the first to apply this newly developed method to this literature. Compared to the existing methods, this approach provides several advantages for modelling health dynamics. Firstly, exploring heterogeneous covariate effects within the quantile regression framework offers a more flexible approach than the classical Gaussian fixed- and random-effects estimators. This is important because certain factors may not only affect the location of the conditional distribution of youth depression, but also affect the scale or other aspect of the distribution. If the underlying mechanism that links these factors with youth depression does differ at different parts of the distribution, using a conditional mean estimation will neglect this and provide different conclusions. Secondly, it is important to separate individual-specific heterogeneity from state dependence when examining the persistence of health outcomes (see Contoyannis et al. 2004a, 2004b); this estimator allows the control of individual-specific effects via fixed effects (allowing for unobserved heterogeneity to be correlated with observed covariates) in the dynamic panel data framework. Thirdly, the proposed model provides a small-T bias-corrected estimate of the true state dependence parameter taking account of the unobserved heterogeneity relative to 'brute force' fixed effects. Lastly, compared to the conditional mean regression models, the proposed estimator will be less sensitive to outliers because it will be less sensitive to the tail behavior of the underlying random variables of interest.

The organization of the paper is as follows. Section 2 describes the data we use. Section 3 presents our proposed modelling approach in details. Section 4 presents the estimation results from both the traditional estimators and our proposed estimator. 
Section 5 provides a concluding discussion.

\section{Description of the Data}

2.1 Data Source and Variable Definitions

This study uses data from the CNLSY79, which is an ongoing biennial longitudinal survey that collects information on the children born to the female respondents of the National Longitudinal Survey of Youth 1979 cohort (NLSY79 Cohort). The NLSY79 Cohort is a longitudinal project that follows the lives of a sample of American youth born between 1957-64; the cohort originally included 12,686 respondents ages 14-22 when first interviewed in 1979. The CNLSY79 began in 1986 and the assessments measure cognitive ability, temperament, motor and social development, and behavior problems of the children and the quality of their home environment. Starting in 1994, children who reach the age of 15 were given the Young Adult survey, which focuses on the transition to adulthood. In 1994, 7,089 children who were born to the 6,283 NLSY79 female respondents were interviewed, among which 6,109 were under age 15 and 980 were 15 years or older. In 2008, 7,660 children were interviewed, among which 1,354 were under age 15 and 6,306 were 15 years or older. We use the respondents of the Young Adult Survey as our study sample. We construct the repeated measures of depression from the Young Adult Survey. We obtain parental variables from the NLSY79 survey, and prenatal and early life variables from the Child Survey.

The outcome variable is a scale of depression-- the Center for Epidemiological Studies Depression Scale (CES-D) developed by Radloff (1977). The CES-D has been used in a large body of studies on depression and has been shown to have high validity and reliability in the general population (Beekman et al. 1997; Weissman et al.1977). Furthermore, the CES-D has been shown to have high internal consistency reliability (i.e. a measure of reliability used to evaluate the degree to which different test items that probe the same construct produce similar results) and test-retest reliability (i.e. a high 
degree of stability over time) for the population of adolescents (Radloff 1991; Roberts et al. 1990). The examination of screening efficacy for the CES-D shows that concurrent validity (i.e. the degree of congruence between the screener and the diagnosis of depression) of the CES-D is reasonably high and consistent across different subpopulations (Lewinsohn et al. 1997). The full-version of CES-D includes 20 questions related to symptoms of depression. In the Young Adult Survey, respondents were given a 7-item, reduced version of the CES-D questionnaire in all waves from 1994 to 2010. Examples of the CES-D questions include: "In the last week I felt that I couldn't shake off the blues, even with help from my family and friends", and "In the last week I felt that everything I did was an effort." The answers to these 7 questions were coded on a scale from 0 to 3 with 0 representing "rarely/none of the time" and 3 representing "most/all of the time". Our study uses the 7-item composite CES-D score (ranging from 0 to 21) as our dependent variable in the analyses. From this point on, we use "the CESD score" to represent the composite score of the 7-item questions.

Our empirical specification includes important observables: age, gender, race, and birth order, prenatal or biological factors, health care utilization, living environment, and family and own SES. Biological factors include age of mother at the birth of the child, mother's drinking and smoking behaviours during pregnancy. We include two variables to capture the effect of health care utilization: whether the youth received help for an emotional problem in the past year, and whether the youth took any medicine or prescription drugs to control behavior in the past year. Variables representing living environment are whether the youth lives in an urban area, and whether the youth lives in a Standard Metropolitan Statistical Area (SMSA).

To capture family SES factors we include maternal education measured as the highest grade completed and maternal employment status measured as the number of weeks unemployed in the past calendar year. We include family income measured as the total net family income, which is adjusted to 2010 dollar equivalents using the Consumer Price Index (CPI) of the interview year. We also include an indicator variable 
for family poverty status because living under the poverty line may contribute to youth depression conditional on the absolute family income level. Finally we include the young adults' employment status variable indicating s/he held a job for two weeks or more in the last year.

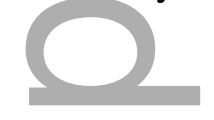

2.2 Study Sample and Descriptive Statistics

7,612 individuals ever completed a Young Adult Survey during the survey years of 1994-2010. We used several criteria to select our sample. First, we only kept the individuals in the Youth Survey who had at least one CES-D measurement during 19942010. Imposing this criterion reduces the sample to 7,598 individuals. Second, we dropped the observations for which an individual was aged 26 or above in any wave of the Young Adults Interview, leading to a further reduction of the sample to 7,541 individuals. Third, we dropped individuals with fewer than three consecutive waves of observation of the CES-D score, because we need to include the first lag of the CES-D score to estimate a dynamic model and the second lag of the dependent variable as the instrumental variable for our IV estimator. After applying this criterion, we have 4,275 individuals with 17,584 observations in the sample. Lastly, we dropped observations with missing values on our main regressors described above; this leaves 3,812 individuals as our study sample with 11,238 observations in total. It is worth noting that the respondents who were dropped are systematically of lower birth order and were born to systematically younger mothers than the included observations. While this may lead us to under or overestimate the overall prevalence of depression in the population, this does not lead to inconsistency in estimation of model parameters as birth order and maternal variables are exogenous in our context.

In Table 1, we list the summary statistics of the variables across all individuals in our study sample and over all waves. About half of the individuals are male with the mean age over all observations around 19. The CES-D depression score has a mean of 
4.5 and a standard deviation of 3.68. About $12.1 \%$ of the observations have CES-D scores of zero. Figure 1 presents a histogram of the CES-D score: the distribution has a long right tail with about $95 \%$ of the values under 12 .

Table 2 presents the transition matrix for the CES-D score classified into five categories: 0, 1-3, 4-6, 7-11 and 12 and above. The categories are chosen to contain relatively equal proportions of the sample. The rows of the transition matrix indicate the depression level in the previous period, while the columns indicate the depression level in the current period. The transition matrix shows that the majority of the transitions among different levels of depression appear on the diagonal or one cell off the diagonal. This indicates that substantial persistence exists in the dynamics of depression for the young adults, with the most persistence observed for those with CES-D scores of 1-3 or 4-6.

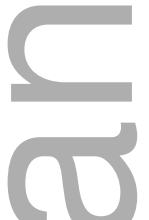

\section{Empirical Methods}

We model dynamics (given the constraints imposed by the data) by including the first lag of the depression score as a regressor. We employ an IV approach suggested in Galvao (2011) for a dynamic quantile regression model with fixed effects as our main empirical approach. We treat the dependent variable as discrete therefore we extend this IV estimator by incorporating the jittering procedure suggested by Machado and Santos Silva (2005). For comparison we first estimate a set of dynamic conditional mean regression models and pooled dynamic quantile regression model-- these models are more conventional therefore only described in the Online Appendix technical note. We describe below our proposed estimation method, i.e. dynamic quantile regression IV model with fixed effects.

We consider a dynamic panel quantile regression model with individual fixedeffects. The $\tau$ th conditional quantile function of the outcome variable of the $t$ th observation on the $i$ th individual $y_{i t}$ can be represented as: 


$$
Q_{y_{i t}}\left(\tau \mid y_{i t-1}, x_{i t}, z_{i}\right)=\alpha(\tau) y_{i t-1}+x_{i t}^{\prime} \beta(\tau)+z_{i} \eta
$$

Where $y_{i t}$ is the outcome of interest, $y_{i t-1}$ is the first lag of the outcome variable, $x_{i t}$ are a set of exogenous variables, $z_{i}$ is the individual indicator and $\eta$ represents the $N x l$ vector of individual-specific effects. The parameter $\alpha$ captures the state dependence level of youth depression. Koenker (2004) developed a regularization method to implement the above fixed-effects model. In this method fixed effects are assumed invariant across quantiles

Galvao (2011) notes that this fixed-effects estimator developed by Koenker (2004) suffers from bias in the presence of lagged dependent variables as regressors when $\mathrm{T}$ is moderate even as $\mathrm{N}$ goes to infinity. Using a rationale analogous to that of Anderson and Hsiao (1981, 1982) and Arellano and Bond (1991), Galvao (2011) suggests that valid instruments for consistently estimating (1) are available within the model. Because the lagged regressors (or functions of them) are correlated with the included regressors but are uncorrelated with the error term, they can be used as instruments. Following Chernozhukov and Hansen (2006, 2008), Galvao (2011) proposed an IV estimator for the state dependence parameter. The implementation of this IV procedure in the context of (1) minimizes the following objective function $R_{N T}$

$$
\begin{gathered}
R_{N T}\left(\tau, \eta_{i}, \alpha, \beta, \gamma\right):=\sum_{k=1}^{K} \sum_{i=1}^{N} \sum_{t=1}^{T} v_{k} \rho_{\tau}\left(y_{i t}-\alpha\left(\tau_{k}\right) y_{i t-1}-x_{i t}^{\prime} \beta\left(\tau_{k}\right)-z_{i} \eta-\right. \\
\left.\omega_{i t}^{\prime} \gamma\left(\tau_{k}\right)\right),
\end{gathered}
$$

where $\rho_{\tau}(u):=u(\tau-I(u<0))$ as in Koenker and Bassett (1978), $v_{k}$ are the weights that control the relative influence of the $K$ quantiles $\left\{\tau_{1}, \ldots, \tau_{K}\right\}$ for estimating the quantile invariant parameters $\eta, \omega_{i t}$ is a $\operatorname{dim}(\gamma)$-vector of instruments such that $\operatorname{dim}(\gamma)>=\operatorname{dim}(\alpha)$ and $\gamma$ is the parameter associated with the instruments. Consistent with the estimator suggested by Koenker (2004), Galvao (2011) restricts the individualspecific effects to be independent of $\tau$ due to the difficulty of estimating $\tau$-dependent individual effects in a short panel of large cross-sections. Specifically, the instruments 
may include values of $y$ lagged two periods or more and/or lags of the exogenous variable $x$ which affect lagged $\mathrm{y}$ but are independent of $u$. The estimator should minimize the effect of $\omega_{i t}$. The intuition is that imposing this restriction is valid when the FE model (1) is the true model and the instruments $\omega_{i t}$ are valid, as $\omega_{i t}$ should be uncorrelated with the error term and should therefore have a zero coefficient at the true values of the parameters.This IV-estimator reduces the bias relative to conventional fixed-effects estimation of the dynamic quantile regression model. Galvao (2011) shows that under some mild regularity conditions, the estimator is consistent and asymptotically normal, while Monte Carlo experiments showed that even in short panels such as ours this IV estimator can substantially reduce bias.

We implement this IV approach suggested by Galvao (2011) to estimate the dynamics of CES-D score. We use the values of CES-D score lagged two periods as our IV, because $y_{i t-2}$ is structurally correlated with $y_{i t-1}$ and this IV is valid under the assumption that the error term is serially uncorrelated conditional on the individual fixed effects. This assumption should be reasonable in this context; however, we are unaware of any tests to formally test this in such model. While we could, under the stronger assumption of predetermined or strict exogeneity of the $\mathrm{X}$ variables, include other period of covariates as instruments, this can be problematic if these are weak instruments (see e.g. Cameron and Trivedi 2005 Ch. 4.9). It is worth nothing that in the case of dynamic conditional mean regression models, the assumption of no serial correlation after controlling for fixed effects is testable if we implement the ArellanoBond type of estimator which employs additional lags of the dependent variables as instruments. This offers an opportunity to implement an overidentifying restrictions test on the validity of the instruments (see Arellano and Bond 1991). In the case of dynamic conditional quantile estimation, however, we are unaware of such tests to test this assumption.

We perform bootstrap-based inference in this context. Specifically, we construct the bootstrap samples by resampling from the cross-sectional units (individual persons 
in our case) with replacement. This is because Monte Carlo simulations suggest that cross-sectional bootstrapping has the best performance among alternative bootstrapping procedures in this context (Galvao and Montes-Rojas 2009; Kato, Galvao and MontesRojas 2012). We used 499 bootstrap replications with a pair-wise resampling technique to construct the empirical distribution of the estimator and construct the bootstrap standard errors. We also used a percentile bootstrap procedure to construct $95 \%$ confidence intervals for the parameters of interest.

Applying the above IV estimator directly to our data is problematic because the CES-D score is not continuous. To address this problem, we apply the IV approach to jittered data of the CES-D score. We first use the jittering process suggested by Machado and Santos Silva (2005) to construct a continuous variable with conditional quantiles that have a one-to-one relationship with the conditional quantiles of the original CES-D score. We then implement the IV estimator with the artificially smoothed CES-D score as the dependent variable (more details are described in Online Appendix). We use 500 jittered samples to derive the estimates for this model and the final estimate takes the average of these estimates. We also experimented with the number of replications, including 50, 100, 500 and 1,000 jittered samples. We chose 500 jittered samples because results change at only the 3rd decimal place when increasing from 500 to 1000 , but the calculation time doubles.

As the jittering process involves a non-linear transformation from the original CES-D score to a smoothed variable, the marginal effect (ME) estimates are different from the coefficient estimates. We use the marginal effects at the mean (MEM) convention to calculate the MEs. According to Equation (1), the MEs for any continuous regressor $x_{j}$ are estimated by $\exp \left(\overline{\boldsymbol{X}}^{\prime} \hat{\beta}\right) \hat{\beta}_{j}$, with all the regressors evaluated at their mean values. For any dummy variable $x_{j}$, we calculate the MEs with respect to a change in this dummy variable from 0 to 1 , using the difference of the corresponding predicted values: $\exp \left({\overline{\boldsymbol{X}_{1}}}^{\prime} \hat{\beta}\right)-\exp \left({\overline{\boldsymbol{X}_{0}}}^{\prime} \hat{\beta}\right)$, where $\overline{\boldsymbol{X}_{1}}$ represents the design matrix 
evaluated at 1 for this dummy variable $x_{j}$ and at the means for all the other regressors, while $\overline{\boldsymbol{X}_{\mathbf{0}}}$ represents the design matrix evaluated at 0 for this dummy variable $x_{j}$ and at the means for all the other regressors. Care is needed in interpreting the results because the quantiles of $y_{i t}$ are step-functions. In particular since $y_{i t}$ is a step function, when the ME is $>1$ for the model for $J_{i t}$ there will be an effect on the quantile of $y_{i t}$; when the ME is $<1$ for $J_{i t}$ it is not necessarily true that there is an effect on the quantile of $y_{i t}$. The paper reports partial effects (evaluated at the mean) on the quantiles of $J_{i t}$, not $y_{i t}$. Practically observing an effect for the jittered sample does not necessarily translate to an impact on the CES-D score if the $\mathrm{ME}<1$.

\section{Estimation Results}

4.1 Results for Dynamic Conditional Mean Regression Models

Table 3 presents results for the dynamic conditional mean regression models using Poisson specification. Columns (1) and (2) present marginal effects and standard errors for the pooled model; columns (3) and (4) present the results for the random-effects model; columns (5) and (6) present the results for the fixed-effects model. Estimates for the pooled model indicate strong positive state dependence. However, the randomeffects model suggests state dependence is statistically insignificant, whereas the fixedeffects model suggests negative state dependence. This "regression to the mean" finding suggests that conditional on other variables and individual fixed effects, a negative serial correlation in depression scores remains. This may be due to negative serial correlation in the errors or negative state dependence. The magnitude of the estimated persistence level based on the fixed-effects model is surprisingly large. However, since the fixed-effects estimator is subject to bias in this context, this estimate needs to be interpreted with caution. Table 3 also show that a group of other covariates have significant inter-temporal effects on youth depression. These include gender, race, psychological health care utilization, age of mother at birth of child, maternal smoking during pregnancy, maternal education and family income. The magnitudes of the effects 
are relatively small-- this is not surprising as the dynamic models only capture the intertemporal effects of these factors conditional on the previous CES-D score rather than long-run effects.

As a comparator we also estimate the pooled, random-effects and fixed-effects models based on a linear specification. In addition we estimated a first-difference (FD) with IV model (Anderson-Hsiao estimator) based on a linear specification, in which the dependent variable lagged for two periods is used as the instrument for the first difference of the lagged dependent variable. These results are only reported in the online appendix (Table A1). Both random-effects and fixed-effects estimators are inconsistent due to the inclusion of lagged dependent variable as a regressor in short panels (see Cameron and Trivedi (2005) Chapter 22). The Anderson-Hsiao estimator is consistent for the state dependence parameter when there is no serial correlation after controlling for individual fixed effects. It is worth nothing that the estimated state dependence based on Anderson-Hsiao estimator is positive but small in magnitude, and is only statistically significant at $10 \%$ level.

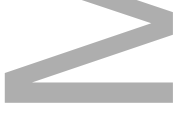

4.2 Results for Dynamic Conditional Quantile Estimation Models

Table 4 presents the conditional quantile estimation results for the pooled model based on 500 jittered samples. Columns (1) and (2) list the marginal effects and the standard errors for the 0.25 conditional quantile of the CSE-D score; Columns (3) and (4) list the results for the 0.5 conditional quantile of the CSE-D score; Columns (5) and (6) present the results for the 0.75 conditional quantile of the CSE-D score. The results show that the estimated persistence level is stronger for larger quantiles of the CES-D distribution. Positive state dependence is stronger at the 0.75 quantile of the conditional CES-D distribution, suggesting high persistence of relatively severe depression. The intertemporal effects of some covariates vary across quantiles of the CES-D score. For example, the gender gap of CES-D score is larger at the 0.75 quantile, while racial 
differences in CES-D scores are smaller at the 0.75 quantile. Maternal tobacco consumption only adversely affects the lower quantiles whereas maternal drinking during pregnancy only adversely affects the highest quantile. Finally, the roles of family SES characteristics differ across quantiles of the CES-D score. The protective effects of higher maternal education and higher family income are larger at the 0.75 quantile.

Table 5 presents the results from our proposed model, i.e. the IV estimator for dynamic conditional quantile regression with individual fixed effects and with jittering. The point estimates of the marginal effects are based on 500 jittered samples. The reported standard errors are based on 499 bootstrap replications. As in the pooled model, the estimated state dependence parameters are positive and the absolute value of the estimated state dependence effect is larger at higher quantiles. However, the state dependence estimate is statistically insignificant for all quantiles. Given that the state dependence estimates from the conditional quantile models without individual fixed effects are statistically significant and positive, this suggests that much of the positive estimated state dependence effect is due to individual fixed effects and that conditional on all the other variables and individual fixed effects, the observed positive state dependence disappears. It is worth noting that instrumenting the first lag of CES-D score with the second lag of the CES-D score leads to a loss of 3,053 observations. This increases the standard errors dramatically. Some different patterns are also observed for other regressors compared to the pooled dynamic quantile regression model results. Consultations for emotional problems and the use of drugs for activity or behaviour problems are still positively associated with higher CES-D scores, but became statistically insignificant after controlling for individual fixed effects. Interestingly, after controlling for the individual fixed effects, both maternal education and family income become statistically insignificant even at the 0.75 quantile.

As a comparator we also estimate the IV estimator for dynamic conditional quantile regression with individual fixed effects but without jittering. The results for this model are only reported in the online appendix (Table A2). Consistent with the main 
results presented in Table 5, the estimated state dependence parameter is positive but statistically insignificant across all estimated quantiles. The magnitudes of the estimates are slightly larger than those based on jittered sample. The patterns with regard to the effect of the other variables remained the same.

\section{Discussion and Conclusion}

This paper has employed a novel statistical approach to model the dynamics of youth depression and to examine the extent of true state dependence conditional on unobserved individual heterogeneity and observed characteristics among a general population of adolescents. We implemented a newly-developed IV quantile regression for dynamic panel with fixed-effects model (Galvao 2011) combined with 'jittering' as suggested by Machado and Santos Silva in the context of estimating count data models with cross-sectional data (Machado and Santos Silva 2005). The results from the dynamic conditional quantile regression models revealed the importance of taking into account time-invariant unobserved heterogeneity when examining the dynamics of youth depression. After taking into account the individual fixed effects, the persistence level of CES-D scores becomes very close to zero across all estimated quantiles. This finding, in conjunction with the positive estimates obtained from the pooled models, suggests that the true state dependence in youth CES-D scores in our study sample is very low and the observed positive association between previous depression and current depression, conditional on observed individual characteristics, is mainly due to timeinvariant unobserved individual heterogeneity. A direct implication is that more sophisticated models and estimators dealing with fixed effects appropriately will lead to different conclusions regarding the importance of previous depression than those standard approaches not allowing for fixed effects. More importantly, our study suggests that the experience of depression per se does not elevate the risk of future depression among the general population during this period of the life course. Given that substantial persistence in depression is attributable to characteristics which do not 
vary over time, identification of what is underlying these individual fixed effects should be given higher priority in order to provide clear policy implications.

The other key finding based on the estimates from the pooled dynamic quantile regression models (without individual fixed effects) is the existence of heterogeneous inter-temporal effects of time-invariant variables, including demographic characteristics and prenatal maternal behaviours, as well as time-varying family SES variables, across quantiles of depression distribution. For example, the gender gap of depression is larger at higher quantiles, while racial differences in depression are smaller at higher quantiles. Given that higher quantiles represent more severe depression, effort should be made to target interventions towards adolescent girls who are at high risk of developing depression. Moreover, maternal smoking during pregnancy only adversely affects the lower quantiles whereas maternal drinking during pregnancy adversely affects the highest quantile. This indicates the importance of focusing on reducing the drinking behaviour of mothers at pregnancy. Unfortunately, the effect of these important timeinvariant variables cannot be identified using a fixed-effects model. The protective effects of higher maternal education and higher family income are larger at the higher quantiles, however these heterogeneous effects disappear when individual fixed effects are included. This again indicates the importance of identifying the underlying reason for time-invariant unobserved heterogeneity.

This study could be improved in a number of ways. Firstly, we would like to estimate these models for high quantiles, e.g. at the 0.9 quantile of the CES-D distribution, because these quantiles would likely capture the individuals who have clinically diagnosed depression and are at risk of very negative outcomes. Unfortunately, we had convergence problems due to sparse data at high quantiles of the distribution. Secondly, if additional waves of data become available we could use multiple lags of the dependent variable and lags of some exogenous covariates as instruments in the estimation of dynamic models and employ tests of overidentifying restrictions. This is currently not feasible given the relatively short T of the CNLSY79 data. Thirdly there 
are some alternative recently proposed approaches to estimating dynamic quantile regression with panel data. Powell (2013) estimates unconditional quantiles-- these give estimates of how the variables of interest impact the unconditional distribution of the outcome-variable rather than the distribution of the outcome variable conditional on all the covariates as given by conditional quantile regression approaches. Arellano and Bonhomme (2013) discuss a random effects quantile regression approach which models the individual effect distribution as a function of initial values and regressors using conditional quantile regression. Chernozhukov et al. (2013) estimate bounds for dynamic quantile models with panel data, arguing that the bounds provide a partialidentification solution to the important problem of estimating the effect of state dependence in the presence of unobserved heterogeneity. The future implementation of these approaches would be useful complements to our analysis.

\section{$\underline{\text { References }}$}

Anderson, T. W., and C. Hsiao (1981) 'Estimation of Dynamic Models with Error Components' Journal of the American Statistical Association, 76, 598-606.

Anderson, T. W., and C. Hsiao (1982) 'Formulation and Estimation of Dynamic Models Using Panel Data' Journal of Econometrics, 18, 47-82.

Arellano, M., and S. Bond (1991) 'Some Tests of Specification for Panel Data: Monte Carlo Evidence and an Application to Employment Equations' Review of Economic Studies, 58, 277-279.

Arellano, M., and S. Bonhomme (2013) 'Random Effects Quantile Regression' Working Paper.

Beekman AT, Deeg DJ, Van Limbeek J, Braam AW, De Vries MZ, Van Tilburg W. (1997) 'Criterion Validity of the Center for Epidemiologic Studies Depression scale (CES-D): results from a community- based sample of older subjects in The Netherlands.' Psychol Med, 27, 231-235.

Cameron A. Colin, and Trivedi Pravin K (2005) 'Microeconometrics:Methods and 


\section{Applications' CAMBRIDGE UNIVERSITY PRESS}

Case, A., Fertig, A and Paxson, C. (2005) 'The lasting impact of childhood health and circumstance' Journal of Health Economics, 24, 365-389

Chernozhukov, V., and C. Hansen (2006) 'Instrumental Quantile Regression Inference for Structural and Treatment Effects Models' Journal of Econometrics, 132, 491-525.

Chernozhukov, V., and C. Hansen (2008) 'Instrumental Variable Quantile Regression: A Robust Inference Approach’ Journal of Econometrics, 142, 379-398.

Chernozhukov, V, Fernández-Val,I, Hahn,J and Newey.W (2013) 'Average and Quantile effects in nonseparable panel models' Econometrica, 81 (2), 535-580

Contoyannis, Paul \& Dooley, Martin (2010) 'The role of child health and economic status in educational, health, and labour market outcomes in young adulthood,' Canadian Journal of Economics, Canadian Economics Association, 43(1), 323-346

Contoyannis, P., Jones, A.M., and Rice, N. (2004a) 'Simulation-based inference in dynamic panel ordered probit models: an application to health' Empirical Economics, $29,49-77$

Contoyannis, P., Jones, A.M., and Rice, N. (2004b) 'The dynamics of health in the British Household Panel Survey' Journal of Applied Econometrics, 19, 473-503

Contoyannis, P., and Li, J. (2011). The evolution of health outcomes from childhood to adolescence. Journal of Health Economics, 30(1), 11-32.

Currie, Janet \& Stabile, Mark, (2006) 'Child mental health and human capital accumulation: The case of ADHD,' Journal of Health Economics, 25(6), 1094-1118

Currie J, Stabile M, Maniyong P, Roos L (2010) 'Child health and young adult outcomes.' Journal of Human Resource, 45, 517-548.

Farb NA, Anderson AK, Bloch RT, Segal ZV. (2011) 'Mood-linked responses in medial prefrontal cortex predict relapse in patients with recurrent unipolar depression.' Biological Psychiatry, 70, 366-372. 
Fergusson, David M.1; Boden, Joseph M.1; Horwood, L. John (2007) 'Recurrence of major depression in adolescence and early adulthood, and later mental health, educational and economic outcomes' British Journal of Psychiatry. ,191(2), 335-342

Galvao, A. (2011) 'Quantile regression for dynamic panel data with fixed effects,' Journal of Econometrics, 164(1), 142-157

Galvao A. F. and Montes-Rojas G. V. (2009) 'A Monte Carlo study on bootstrap inference for penalized quantile regression panel data,' Unpublished manuscript, 2009

Goodman, A., Joyce, R., \& Smith, J. P. (2011) 'The long shadow cast by childhood physical and mental problems on adult life.' Proceedings of the National Academy of Sciences, 108(15), 6032-6037.

Greden J.(2001) 'The burden of recurrent depression: causes consequences, and future prospects' Journal of Clinical Psychiatry, 62(Suppl. 22), 5-9.

Ham, J. C., Iorio, D., and Sovinsky, M. (2013) 'Caught in the Bulimic Trap? Persistence and State Dependence of Bulimia Among Young Women.' Journal of Human Resources, 48(3), $736-767$.

Hauck, K., and Rice, N. (2004) 'A longitudinal analysis of mental health mobility in Britain.' Health economics, 13(10), 981-1001.

Heckman, J. J. (2012). 'The developmental origins of health.' Health economics, 21(1), 24-29.

Hsiao. C (2003) 'Analysis of Panel Data' $2^{\text {nd }}$ edition. Cambridge Univerity Press. Jones, A.M., Rice, N., Contoyannis, P., (2012) 'The dynamics of health', In: The Elgar Companion to Health Economics. $2^{\text {nd }}$ edition. Edward Elgar Publishing Limited, Chapter 2.

Kato, K., Galvao, A. F., and Montes-Rojas, G. V. (2012) 'Asymptotics for panel quantile regression models with individual effects.' Journal of Econometrics, 170(1), 76-91.

Koenker, R., and G. W. Bassett (1978) 'Regression Quantiles' Econometrica, 46, 33-49. 
Koenker, R., (2004) 'Quantile regression for longitudinal data,' Journal of Multivariate Analysis, Elsevier, 91(1), 74-89.Lewinsohn PM, and Essau CA, (2002) 'Depression in adolescents.' Guilford Press

Lewinsohn PM, Rhode P, Klein DN, Seeley JR. (1999) 'Natural course of adolescent major depressive disorder, I: continuity into young adulthood.' J Am Acad Child Adolesc Psychiatry, 38, 56-63.

Lewinsohn, Peter M., Seeley, John R., Roberts, Robert E., Allen, Nicholas B. (1997) 'Center for Epidemiologic Studies Depression Scale (CES-D) as a screening instrument for depression among community-residing older adults.' Psychology and Aging, 12(2), $277-287$.

Machado, J.A.F., and Santos Silva, J. M. C., (2005) 'Quantiles for Counts,' Journal of the American Statistical Association, American Statistical Association, 100, 1226-1237.

Merikangas KR, He JP, Burstein M, Swanson SA, Avenevoli S, Cui L, Benjet C, Georgiades K, Swendsen J. (2010) 'Lifetime Prevalence of Mental Disorders in U.S. Adolescents: Results from the National Comorbidity Survey Replication-Adolescent Supplement (NCS-A).' J Am Acad Child Adolesc Psychiatry. 49(10), 980-989.

Powell, D. (2013) ‘A New Framework for Estimation of Quantile Treatment Effects: Nonseparable Disturbance in the Presence of Covariates', RAND Corporation Publications Department. Working paper WR-824-1

Radloff, Lenore S. (1977) 'The CES-D Scale: A Self-Report Depression Scale for Research in the General Population.' Applied Psychological Measurement, 1, 385-401.

Radloff, Lenore S. (1991) 'The use of the Center for Epidemiologic Studies Depression Scale in adolescents and young adults.' Journal of Youth and Adolescence, 20(2), 149166

Repetto, P., Caldwell, C., and Zimmerman, M. (2004) 'Trajectories of depressive symptoms among high risk African-American adolescents' Journal of Adolescence, 
26(2), 169-183

Roberts, Robert E.; Andrews, Judy A.; Lewinsohn, Peter M.; Hops, Hyman (1990) 'Assessment of depression in adolescents using the Center for Epidemiologic Studies Depression Scale.' Psychological Assessment: A Journal of Consulting and Clinical Psychology, 2(2), 122-128.

Roy, J., and Schurer, S. (2013) 'Getting Stuck in the Blues: Persistence of Mental Health Problems in Australia.' Health economics, 22(9), 1139-1157.

Rushton, J., Forcier, M., and Schectman, R. (2002) 'Epidemiology of Depressive Symptoms in the National Longitudinal Study of Adolescent Health' Journal of the American Academy of Child \& Adolescent Psychiatry, 41(2), 199-205.

Smith, James. (2009) 'The Impact of Childhood Health on Adult Labor Market Outcomes.' The Review of Economics and Statistics, 91(3):478-89

Smith JP, Smith GC (2010) 'Long-term economic costs of psychological problems during childhood.' Social Science \& Medicine, 71, 110-115.

Strohschein, Lisa (2005) 'Household income histories and child mental health trajectories' Journal of Health and Social Behavior, 46 (4), 359-375.

Weissman MM, Sholomskas D, Pottenger M, Prusoff BA, Locke BZ. (1977) 'Assessing depressive symptoms in five psychiatric populations: a validation study.' Am $J$ Epidemiol, 106, 203-214.

Weissman MM, Wolk S, Goldstein RB, et al. (1999) 'Depressed adolescents grown up.' JAMA, 281, 1707-1713.

Table 1. Descriptive statistics of variables used for estimation 


\begin{tabular}{|c|c|c|c|}
\hline Variables & Mean & Std. dev. & Median \\
\hline Youth CES-D depression score & 4.496 & 3.679 & 4 \\
\hline Youth CES-D=0 & $12.14 \%$ & & \\
\hline Youth Sex (Male) & $49.00 \%$ & & \\
\hline \multicolumn{4}{|l|}{ Youth race } \\
\hline Hispanic & $22.84 \%$ & & \\
\hline Black & $34.40 \%$ & & \\
\hline Non-Hispanic, non-black & $42.76 \%$ & & \\
\hline \multicolumn{4}{|l|}{ Birth order of youth } \\
\hline First & $39.93 \%$ & & \\
\hline Secon & $34.65 \%$ & & \\
\hline Third & $16.99 \%$ & & \\
\hline Fourth and above & $8.42 \%$ & & \\
\hline Youth live in urban area $(0-1)$ & 0.780 & 0.414 & 1 \\
\hline Youth live in SMSA (0-1) & 0.888 & 0.315 & 1 \\
\hline Youth has a job for 2 weeks or more $(0-1)$ & 0.711 & 0.453 & 1 \\
\hline Youth emotional problem in last year $(0-1)$ & 0.072 & 0.259 & 0 \\
\hline Youth prescription drug for behavior problem (0-1) & 0.039 & 0.194 & 0 \\
\hline Age of mother at birth of child & 24.567 & 3.752 & 25 \\
\hline Mother drinking alcohol during pregnancy $(0-1)$ & 0.431 & 0.495 & 0 \\
\hline Mother smoking during pregnancy $(0-1)$ & 0.307 & 0.461 & 0 \\
\hline Highest grade completed by mother & 12.710 & 2.567 & 12 \\
\hline $\begin{array}{l}\text { Maternal \# of weeks unemployed in past calendar } \\
\text { year }\end{array}$ & 2.254 & 8.657 & 0 \\
\hline Total real annual family income (in 2010 dollars) & $65,528.6$ & $67,661.62$ & $50,479.29$ \\
\hline $\begin{array}{l}\text { Poverty status of mother's family in past calendar } \\
\text { year (0-1) }\end{array}$ & 0.212 & 0.408 & 0 \\
\hline
\end{tabular}

Figure 1. Histogram of youth CES-D depression score 


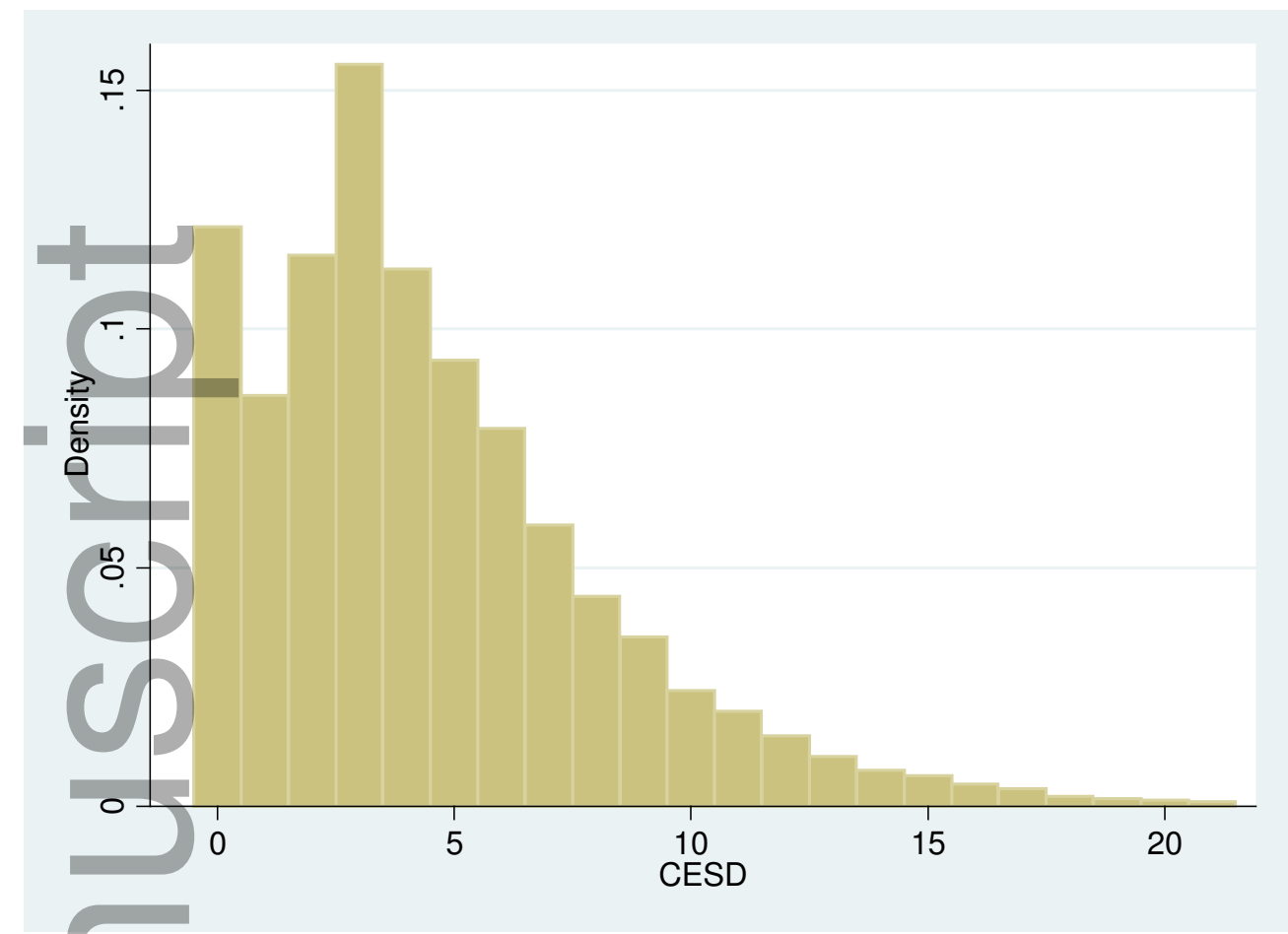

Table 2. Transition matrix for the CES-D score over all waves (5 categories)

\begin{tabular}{|c|c|c|c|c|c|c|}
\hline & \multicolumn{5}{|c|}{ CES-D $_{t}$} & \multirow[b]{2}{*}{ Total } \\
\hline & 0 & $1-3$ & $4-6$ & $7-11$ & $12-21$ & \\
\hline $\mathrm{CES}_{\mathrm{D}-1} \mathrm{D}_{\mathrm{t}}$ & 29.42 & 41.63 & 19.59 & 7.53 & 1.82 & 100 \\
\hline CES-D & 15.18 & 43.4 & 27.54 & 11.66 & 2.22 & 100 \\
\hline CES-D ${ }_{t}$ & 8.36 & 34.03 & 34.64 & 19.59 & 3.39 & 100 \\
\hline CES-D ${ }_{t-1}$ & 5.41 & 21.82 & 31.85 & 30.1 & 10.82 & 100 \\
\hline CES-D $_{t-1} \quad 12-21$ & 4.55 & 15.86 & 21.51 & 34.54 & 23.55 & 100 \\
\hline Tot & & & & & & 100 \\
\hline
\end{tabular}

Note: the cells represent the unconditional transition probabilities in percentages. The bold cell shows the biggest cell in each row. 
Table 3. Dynamic conditional mean regression models-- Poisson Specification

\begin{tabular}{|c|c|c|c|c|c|c|}
\hline & (1) & (2) & (3) & (4) & (5) & (6) \\
\hline \multirow[t]{2}{*}{$\sqrt{-1}$} & \multicolumn{2}{|c|}{ Poisson specification } & \multicolumn{2}{|c|}{ Poisson specification } & \multicolumn{2}{|c|}{ Poisson specification } \\
\hline & Marg. Eff. & St. Err. & Marg. Eff. & St. Err. & Marg. Eff. & St. Err. \\
\hline CESDlag (t-1) & $0.2747 * * *$ & 0.0091 & -0.0139 & 0.0124 & $-0.0431 * * *$ & 0.0088 \\
\hline Youth Gender: male & $-0.3603 * * *$ & 0.0732 & $-0.6797 * * *$ & 0.0979 & & \\
\hline Race: black & $0.2701 * * *$ & 0.1022 & $0.4197 * * *$ & 0.1343 & & \\
\hline $\begin{array}{l}\text { Race: non-Hispanic \& } \\
\text { non-black }\end{array}$ & -0.0349 & 0.1044 & -0.0170 & 0.1346 & & \\
\hline Birth order 2 & 0.1348 & 0.0874 & $0.2472 * *$ & 0.1157 & & \\
\hline Birth order3 & 0.1365 & 0.1116 & 0.2155 & 0.1449 & & \\
\hline Birth order4 & 0.1698 & 0.1454 & 0.3130 & 0.1984 & & \\
\hline $\begin{array}{l}\text { Emotional problem } \\
\text { consultation last year }\end{array}$ & $0.9936^{* * *}$ & 0.1683 & $0.8528 * * *$ & 0.1760 & $0.0833 * *$ & 0.0408 \\
\hline $\begin{array}{l}\text { Drug use for behavior } \\
\text { problem last year }\end{array}$ & $1.0348 * * *$ & 0.2195 & $1.0896 * * *$ & 0.2461 & $0.1407 * *$ & 0.0647 \\
\hline Youth has a job & -0.0242 & 0.0889 & 0.0280 & 0.1016 & 0.0311 & 0.0240 \\
\hline $\begin{array}{l}\text { Age of mother at birth } \\
\text { of child }\end{array}$ & $-0.0254 * *$ & 0.0112 & $-0.0444 * * *$ & 0.0146 & & \\
\hline $\begin{array}{l}\text { Mother drinking } \\
\text { during pregnancy }\end{array}$ & 0.1275 & 0.0778 & 0.1566 & 0.1040 & & \\
\hline $\begin{array}{l}\text { Mother smoking } \\
\text { during pregnancy }\end{array}$ & $0.2644 * * *$ & 0.0843 & $0.4858 * * *$ & 0.1077 & & \\
\hline Youth living in urban & 0.0693 & 0.0975 & 0.0610 & 0.1127 & 0.0080 & 0.0305 \\
\hline $\begin{array}{l}\text { Youth living in } \\
\text { SMSA }\end{array}$ & -0.0475 & 0.1344 & 0.1191 & 0.1686 & $0.1109 *$ & 0.0657 \\
\hline $\begin{array}{l}\text { Maternal highest } \\
\text { grade completed }\end{array}$ & $-0.0411 * *$ & 0.0170 & $-0.0491 * *$ & 0.0240 & -0.0058 & 0.0113 \\
\hline $\begin{array}{l}\text { Maternal \# of weeks } \\
\text { unemployed last year }\end{array}$ & 0.0005 & 0.0043 & 0.0039 & 0.0048 & 0.0014 & 0.0012 \\
\hline $\begin{array}{l}\text { Maternal total family } \\
\text { income* }\end{array}$ & $-0.0013 * *$ & 0.0006 & $-0.0013 *$ & 0.0007 & -0.0001 & 0.0002 \\
\hline $\begin{array}{l}\text { Maternal family } \\
\text { poverty status }\end{array}$ & 0.1149 & 0.0978 & 0.0879 & 0.1269 & -0.0136 & 0.0347 \\
\hline
\end{tabular}


1. *Maternal family income is CPI inflated according to the interview year and the value is in 1000 US dollars.

2. The reported standard errors are robust to cluster effects for the pooled specification.

3. $* * *$ denotes statistical significance at $1 \%$ level, $* *$ denotes statistical significance at $5 \%$ level, * denotes statistical significance at $10 \%$ level.

4. The time-invariant regressors are automatically dropped from the fixed-effects model.

Table 4. Pooled Dynamic Conditional Quantile regression models with jittering

\begin{tabular}{|c|c|c|c|c|c|c|}
\hline & (1) & (2) & (3) & (4) & $(5)$ & (6) \\
\hline & \multicolumn{2}{|c|}{ 0.25 Quantile regression } & \multicolumn{2}{|c|}{0.50 Quantile regression } & \multicolumn{2}{|c|}{ 0.75 Quantile regression } \\
\hline & Marg. Eff. & St. Err. & Marg. Eff. & St. Err. & Marg. Eff. & St. Err \\
\hline CESDlag (t-1) & $0.2068 * * *$ & 0.0091 & $0.2975 * * *$ & 0.0094 & $0.3919 * * *$ & 0.0160 \\
\hline Youth Gender: ma & -0.1066 & 0.0820 & $-0.2966 * * *$ & 0.0728 & $-0.5683 * * *$ & 0.1193 \\
\hline Race: black & $0.3091 * *$ & 0.1209 & $0.3189 * * *$ & 0.1054 & 0.2549 & 0.1598 \\
\hline $\begin{array}{l}\text { Race: non-Hispanic } \\
\text { non-black }\end{array}$ & 0.0274 & 0.1242 & 0.0539 & 0.1068 & 0.0145 & 0.1551 \\
\hline Birth order2 & 0.1634 & 0.1023 & 0.1205 & 0.0854 & 0.1109 & 0.1274 \\
\hline Birth order3 & $0.2354 *$ & 0.1210 & 0.0486 & 0.1195 & 0.2432 & 0.1797 \\
\hline Birth order4 & 0.1320 & 0.1713 & 0.0302 & 0.1457 & 0.3407 & 0.2808 \\
\hline $\begin{array}{l}\text { Emotional problem } \\
\text { consultation last year }\end{array}$ & $0.7517 * * *$ & 0.1638 & $0.9270 * * *$ & 0.1764 & $1.0819 * * *$ & 0.2780 \\
\hline $\begin{array}{l}\text { Drug use for behav } \\
\text { problem last year }\end{array}$ & $0.7485 * * *$ & 0.1833 & $1.0007 * * *$ & 0.2081 & $1.2275 * * *$ & 0.4087 \\
\hline Youth has a job & 0.0725 & 0.1042 & -0.0964 & 0.0915 & 0.0064 & 0.1630 \\
\hline $\begin{array}{l}\text { Age of mother at birth of } \\
\text { child }\end{array}$ & -0.0141 & 0.0125 & -0.0173 & 0.0114 & $-0.0398 * *$ & 0.0176 \\
\hline $\begin{array}{l}\text { Mother drinking during } \\
\text { pregnancy }\end{array}$ & $-0.2124 * *$ & 0.0840 & 0.0036 & 0.0786 & $0.3309 * * *$ & 0.1190 \\
\hline $\begin{array}{l}\text { Mother smoking during } \\
\text { pregnancy }\end{array}$ & $0.3257 * * *$ & 0.0984 & $0.2716 * * *$ & 0.0856 & 0.2035 & 0.1327 \\
\hline Youth living in urban & 0.0991 & 0.1066 & 0.0169 & 0.1010 & $0.2310 *$ & 0.1360 \\
\hline
\end{tabular}




$\begin{array}{lcccccc}\begin{array}{l}\text { Youth living in SMSA } \\ \text { Maternal highest grade }\end{array} & -0.0959 & 0.1445 & -0.0513 & 0.1421 & -0.2784 & 0.2176 \\ \begin{array}{l}\text { completed } \\ \text { Maternal \# of weeks }\end{array} & -0.0256 & 0.0192 & -0.0424 * * & 0.0176 & -0.0613 * * & 0.0242 \\ \begin{array}{l}\text { unemployed last year } \\ \text { Maternal total family }\end{array} & -0.0067 & 0.0053 & 0.0016 & 0.0049 & 0.0078 & 0.0088 \\ \begin{array}{l}\text { income* } \\ \text { Maternal family poverty }\end{array} & -1.00 \mathrm{E}-03 & 6.89 \mathrm{E}-04 & -1.11 \mathrm{E}-03 & 6.76 \mathrm{E}-04 & -2.31 \mathrm{E}-03 * * * & 7.47 \mathrm{E}-04 \\ \text { status } & 0.0574 & 0.1065 & 0.1349 & 0.1080 & 0.1397 & 0.1486\end{array}$

1. * Maternal family income is CPI inflated according to the interview year and the value is in 1000 US dollars.

2. All the estimates are based on 500 jittering replications.

3. The marginal effects are calculated based on the jittered sample.

4. $* * *$ denotes statistical significance at $1 \%$ level, ** denotes statistical significance at $5 \%$ level, * denotes statistical significance at $10 \%$ level.

Table 5. Dynamic conditional quantile regression models: instrumental variable approach with individual fixed effects and jittering

\begin{tabular}{|c|c|c|c|c|c|c|}
\hline & (1) & (2) & (3) & (4) & (5) & (6) \\
\hline & \multicolumn{2}{|c|}{ 0.25 Quantile regression } & \multicolumn{2}{|c|}{ 0.50 Quantile regression } & \multicolumn{2}{|c|}{0.75 Quantile regression } \\
\hline & Marg. Eff. & St. Err. & Marg. Eff. & St. Err. & Marg. Eff. & St. Err. \\
\hline CESDlag (t-1) & 0.0169 & 0.0163 & 0.0403 & 0.0358 & 0.0568 & 0.0591 \\
\hline Youth Gender: male & & & & & & \\
\hline Race: black & & & & & & \\
\hline Race: non-Hispanic & & & & & & \\
\hline non-black & & & & & & \\
\hline Birth order2 & & & & & & \\
\hline Birth order3 & & & & & & \\
\hline Birth orde & & & & & & \\
\hline $\begin{array}{l}\text { Emotional problem } \\
\text { consultation last year }\end{array}$ & 0.0185 & 0.0593 & 0.0300 & 0.1370 & 0.0110 & 0.1457 \\
\hline $\begin{array}{l}\text { Drug use for behavior } \\
\text { problem last year }\end{array}$ & 0.1624 & 0.1148 & 0.3265 & 0.2558 & 0.3149 & 0.2922 \\
\hline
\end{tabular}


Youth has a job

$-0.0633$

0.0549

$-0.1059$

0.1107

$-0.1056$

0.1127

Age of mother at birth of

child

Mother drinking during

pregnancy

Mother smoking during

pregnancy

Youth living in urban

Youth living in SMSA

0.0589

0.0155

0.1318

$-0.0714$

0.1619

$-0.1434$

0.1677

$-0.4452$

0.3694

$-0.2521$

0.3572

Maternal highest grade

completed

$-0.009$

0.0168

$-0.0059$

0.0343

$-0.0218$

0.0464

Maternal \# of weeks

unemployed last year

$-0.0005$

0.0027

$-0.0042$

0.0058

$-0.0036$

0.0058

Maternal total family

income*

$-0.0004$

0.0005

$-0.0005$

0.0011

$-0.0002$

0.0009

Maternal family poverty

status

$-0.0297$

0.0668

$-0.0935$

0.1344

$-0.1711$

0.1299

Constant

$0.9386 * * *$

0.2909

$2.3555 * * *$

0.5798

$2.5120 * * *$

0.7104

1. * Maternal family income is CPI inflated according to the interview year and the value is in 1000 US dollars.

2. The point estimates of the marginal effects are based on 500 jittering replications.

3. The reported standard errors are based on 499 bootstrapping replications.

4. $* * *$ denotes statistical significance at $1 \%$ level, ** denotes statistical significance at $5 \%$ level, * denotes statistical significance at $10 \%$ level.

5. The time-invariant regressors are dropped from the fixed-effects model.

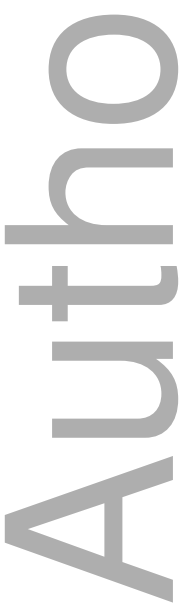




\section{University Library}

\section{- M M I N E R VA A gateway to Melbourne's research publications}

Minerva Access is the Institutional Repository of The University of Melbourne

\section{Author/s:}

Contoyannis, $\mathrm{P} ; \mathrm{Li}, \mathrm{J}$

Title:

The dynamics of adolescent depression: an instrumental variable quantile regression with fixed effects approach

Date:

2017-06-01

\section{Citation:}

Contoyannis, P. \& Li, J. (2017). The dynamics of adolescent depression: an instrumental variable quantile regression with fixed effects approach. JOURNAL OF THE ROYAL STATISTICAL SOCIETY SERIES A-STATISTICS IN SOCIETY, 180 (3), pp.907-922. https:// doi.org/10.1111/rssa.12225.

Persistent Link:

http://hdl.handle.net/11343/291648 